\title{
Portable pathogen diagnostics using microfluidic cartridges made from continuous liquid interface production additive manufacturing
}

Jacob Berger ${ }^{1,2^{*}}$, Mehmet Y. Aydin ${ }^{3^{*}}$, Robert Stavins ${ }^{3}$, John Heredia ${ }^{1,2}$, Enrique Valera ${ }^{1,2}$, Rashid Bashir ${ }^{1,2,3,4 \dagger}$, and William P. King ${ }^{2,3,4 \dagger}$

${ }^{1}$ Department of Bioengineering; ${ }^{2}$ Holonyak Micro and Nano Technology Laboratory, ${ }^{3}$ Department of Mechanical Science and Engineering, ${ }^{4}$ Carle Illinois College of Medicine, University of Illinois Urbana-Champaign, Urbana, IL 61801

*These authors contributed equally to this work

†Towhom correspondence may be addressed: wpk@illinois.edu, rbashir@illinois.edu

\section{Lysing Buffer Compositions:}

The lysis buffer compositions are:

Saponin lysis buffer ${ }^{1}: 0.12 \%$ Formic acid $(v / v)$ and $0.05 \%$ Saponin $(w / v)$ in DI water.

ACK lysis buffer: $155 \mathrm{mM}$ Ammonium Chloride, 9.9mM Potassium Bicarbonate and 0.1mM EDTA in DI water.

SDS $^{2,3}$ Lysis buffer: The composition of lysing solution is, $35 \mathrm{mM}$ sodium dodecyl sulfate (SDS)

Triton-X 1004: Lysis buffer: 1\% (v/v) TritonX-100 in DI water

\section{DNA and Bacterial Culture Sample Preparation}

Pathogen strain for Escherichia Coli, Genomic DNA of Escherichia Coli (O157:H7) and Methicillin Resistant Staphylococcus Aureus (MRSA) strain HFH - 30106, NR-10320, and Staphylococcus aureus (MSSA), Strain MN8, HM-162D were obtained from BEI Resources. These genomic DNA vials were aliquoted and stored at $-80^{\circ} \mathrm{C}$. Appropriate stock volumes were used either for direct experimentation or diluted to the right concentration in buffer or whole blood. For experiments using whole pathogenic bacteria, E. coli (O157:H7), MRSA, strain HFH - 30106, NR-10192,

Staphylococcus aureus (MSSA), Strain MN8, HM-162D were obtained through BEI Resources. Media and agar plates for culture growth and counting were obtained from the Cell Media Facility at the University of Illinois Urbana-Champaign. Luria-Bertani (LB) broth/agar and Tryptic Soy broth/agar (TSB) were used for $E$. coli and MRSA culture respectively. Bacteria were incubated and grown in their respective broth at $37^{\circ} \mathrm{C}$ for 16 hours overnight after which PBS stocks were prepared. PBS stocks of pathogens were prepared based on a reported protocol ${ }^{5}$. $250 \mu \mathrm{L}$ of the 16 -hour cultured broth was centrifuged at $5000 \mathrm{~g}$ for $10 \mathrm{~min}$, and the resulting pellet was washed $2 \mathrm{x}$ in PBS before resuspending the final stock in PBS. Stock dilutions were done to the correct concentration and plated to determine each stocks concentration of pathogens. Single donor human whole blood with K2 anticoagulant (Innovative Research) was purchased and stored at $4{ }^{\circ} \mathrm{C}$ on a rotator. Blood was aliquoted and spiked with pathogens directly before experiments. 


\section{Portable Diagnostic Module Fabrication}

A smartphone (Huawei P30 Pro, Huawei) detects the fluorescence emission from on-chip LAMP assays. The main parts of the system include optical, electrical, and heating components. The smartphone aligns its camera with a macro lens (12.5x, Techo-Lens-01, Techo) and long pass filter (525 nm,84-744, Edmund Optics) which only allows emission from the signal dye into the camera. A printed circuit board (PCB) controls the illumination of eight LEDs ( $\lambda$ peak $=485 \mathrm{~nm}$, XPEBBL, Cree) mounted in a circle to provide uniform illumination. which are covered with short-pass filters (490 nm,490SP Rapid Edge, Omega Filters). The cradle contains two separate on-off switches to control the PCB and a heater. The cartridge is placed on a heating element for 50 minutes at $65^{\circ} \mathrm{C}$. The smartphone recorded images of the cartridge at 5-min intervals during the first 40 min of incubation, then at 2-min intervals to record amplification as it passes the threshold. Smartphone images were recorded with camera settings $($ ISO $)=1000$ and exposure time $=1.0 \mathrm{~s}$.

\section{References:}

1 U. Hassan, T. Ghonge, B. Reddy, M. Patel, M. Rappleye, I. Taneja, A. Tanna, R. Healey, N. Manusry, Z. Price, T. Jensen, J. Berger, A. Hasnain, E. Flaugher, S. Liu, B. Davis, J. Kumar, K. White and R. Bashir, Nat. Commun., 2017, 8, 15949.

2 R. B. Brown and J. Audet, J. R. Soc. Interface, 2008, 5, S131.

$3 \quad$ M. S. Islam, A. Aryasomayajula and P. R. Selvaganapathy, Micromachines, 2017, 8.

4 M. Mahalanabis, H. Al-Muayad, M. D. Kulinski, D. Altman and C. M. Klapperich, Lab Chip, 2009, 9, 2811-2817.

5 C. H. Liao and L. M. Shollenberger, Lett. Appl. Microbiol., 2003, 37, 45-50. 


\section{Supplemental Figures:}

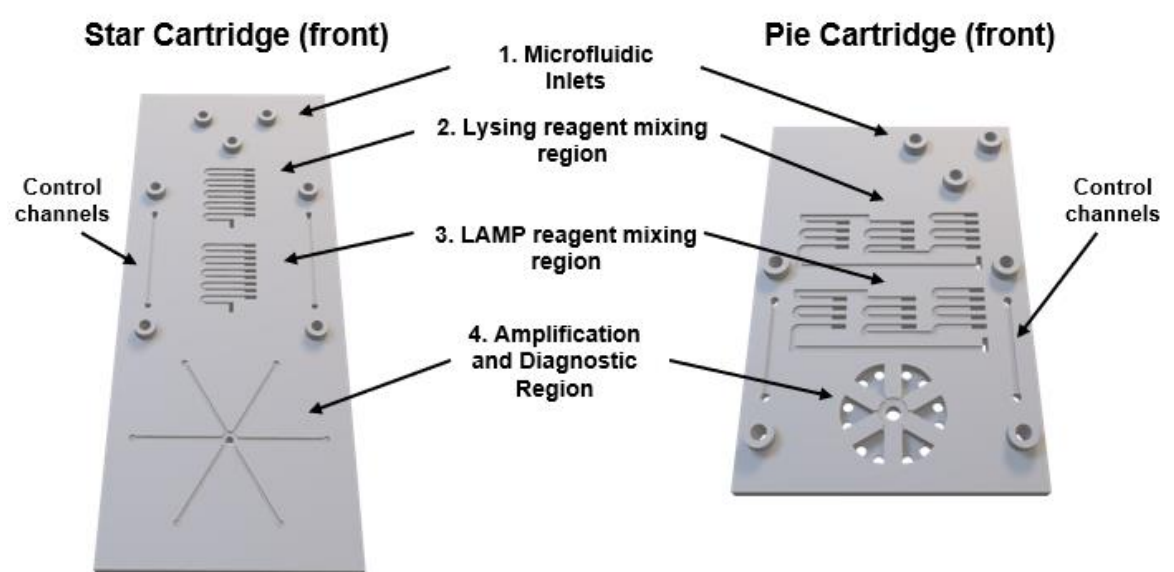

Star Cartridge (back)

Pie Cartridge (back)
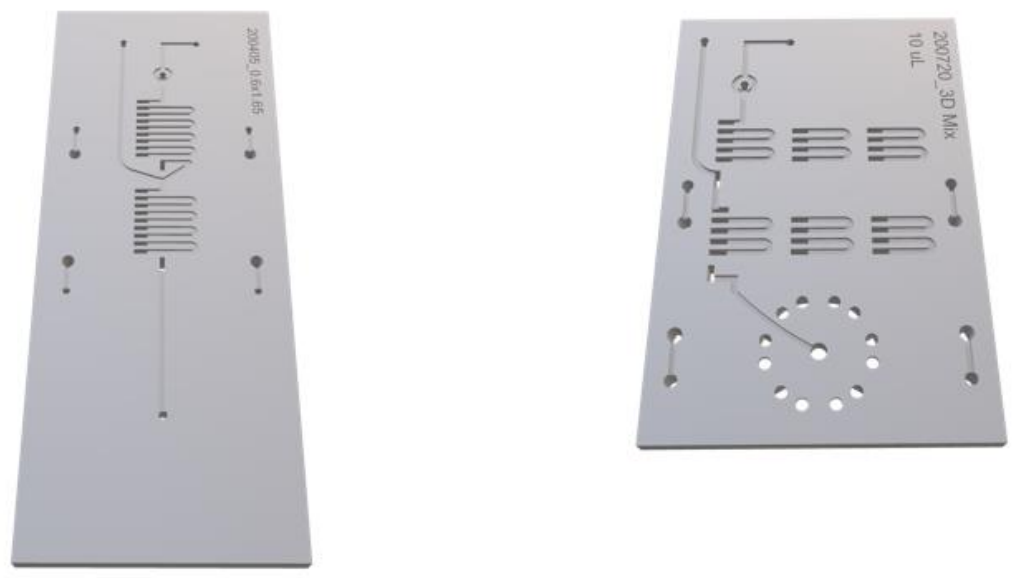

Fig S1. Design features for performing the developed LAMP assay on the diagnostic device.

\section{Diagnostic Device Dimension Details:}

The device is designed with microfluidic inlets with an internal diameter that tapers from 1.65 $\mathrm{mm}$ to $0.90 \mathrm{~mm}$, allowing for an interference fit with $1.6 \mathrm{~mm}$ diameter PTFE (Polytetrafluoroethylene) tubes inserted into the device. The cartridge surface is covered with transparent biocompatible tape (ARSeal 90880, Adhesive Research). The tape transparency allows for optical imaging of the device during filling and detection. The device architecture having channels configured on the surfaces and sealed with tape is appropriate for resin-based additive processes, as the device design must allow for excess resin to be cleaned from the channels in post-processing.

In the first cartridge, with the star point detection region, the blood sample and lysing reagents flow together through the first micromixing region, where the flow takes a vertical turn from one face to the other face between each horizontal U-turn. The lysed sample is then joined by the LAMP reagents with a Y-shaped channel geometry. The combined flow then goes through the second mixing region. Each mixer set has four serpentines on the bottom face and five on the top face, with each serpentine microchannel being $0.7 \mathrm{~mm}$ wide, $0.4 \mathrm{~mm}$ deep, and $8 \mathrm{~mm}$ long. The alternating horizontal and vertical U-turns enhance mixing and allow for dense packing of the mixing structure. The fluid then flows into a detection region consisting of six channels with circular regions on the end arranged in a star pattern. Each channel is $0.5 \mathrm{~mm}$ wide, $0.5 \mathrm{~mm}$ deep, and $10.7 \mathrm{~mm}$ long, while the circular regions on the end have a diameter of $0.88 \mathrm{~mm}$ and are $0.5 \mathrm{~mm}$ deep. Additionally, on the two long edges of the cartridge there are control channels. Each control channel is $0.5 \mathrm{~mm}$ wide, $0.4 \mathrm{~mm}$, deep and $15.15 \mathrm{~mm}$ long 
and are connected to inlets by rectangular channels on the opposing face that are $0.5 \mathrm{~mm}$ wide, 0.4 $\mathrm{mm}$ deep, and $2.9 \mathrm{~mm}$ long.

In the cartrige with the pie reservoir detection region, similarly the sample and flow to the first mixer set. After the first mixing region the fluid is brought back to join the LAMP reagents through a long vertical channel. The fluid then goes through the second mixing region. For this chip each mixing region consists of three vertically stacked sets of serpentine channels. Each of these three sets has two serpentine channels on the bottom face and on the top face there are two full serpentine channels and then a half serpentine channel which then connects to the next set. The serpentine channel dimensions are $0.6 \mathrm{~mm}$ wide, $0.4 \mathrm{~mm}$ deep, and $6 \mathrm{~mm}$ long. Finally, the fluid flows into six reservoirs that radially surround the flow channel furcation. These detection reservoirs located at the end of the cartridge are designed to contain a volume of $\sim 10 \mu \mathrm{L}$ per chamber. The amplification chambers have a $0.5-\mathrm{mm}$-thick wall and two 1.1-mm-diameter outlet holes to remove excess air during filling. Again, there are two control channels on each of the long edges of the cartridge. The control channels are 0.5 $\mathrm{mm}$ wide, $0.4 \mathrm{~mm}$ deep, and $10 \mathrm{~mm}$ long and are connected to the inlets by $0.5 \mathrm{~mm}$ wide, $0.4 \mathrm{~mm}$ deep, and $2.85 \mathrm{~mm}$ long channels.

a

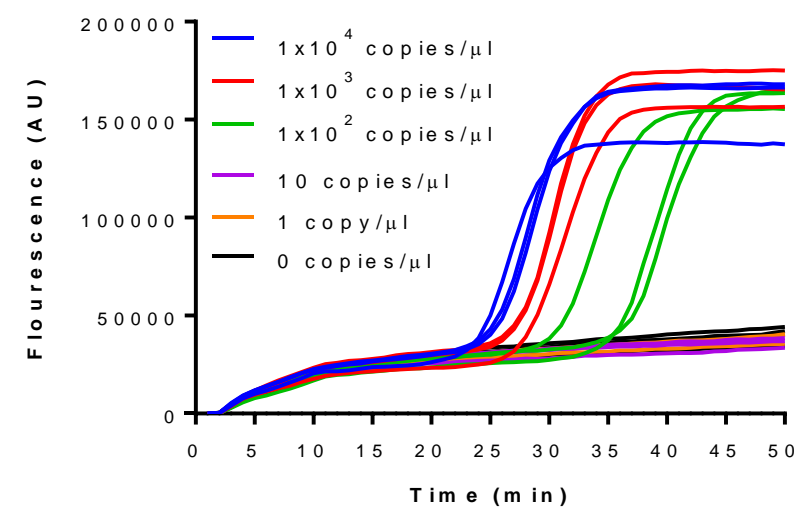

b

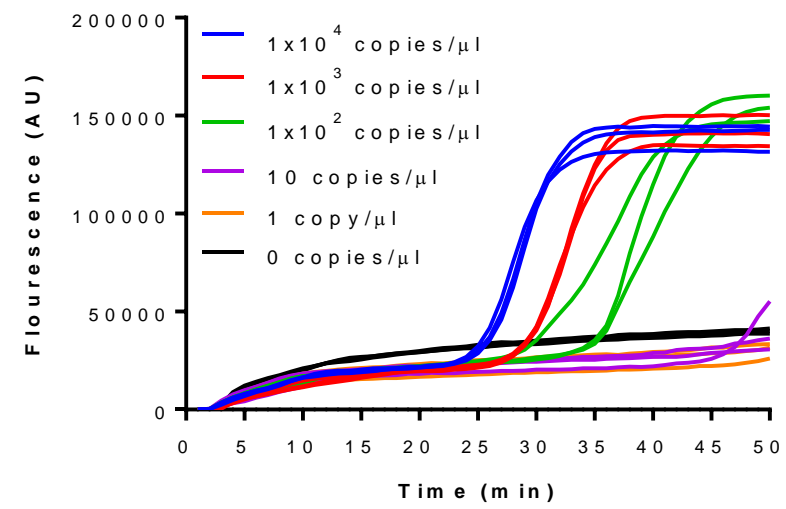

Fig S2. (a) Baseline-subtracted raw fluorescence amplification curves $(n=3)$ across a standard curve for the detection of E. coli DNA. (b) Baseline-subtracted raw fluorescence amplification curves $(n=3)$ across a standard curve for the detection of E. coli DNA with $1 \times 10^{4}$ copies/ $\mu$ l off target MRSA/MSSA DNA in background. 


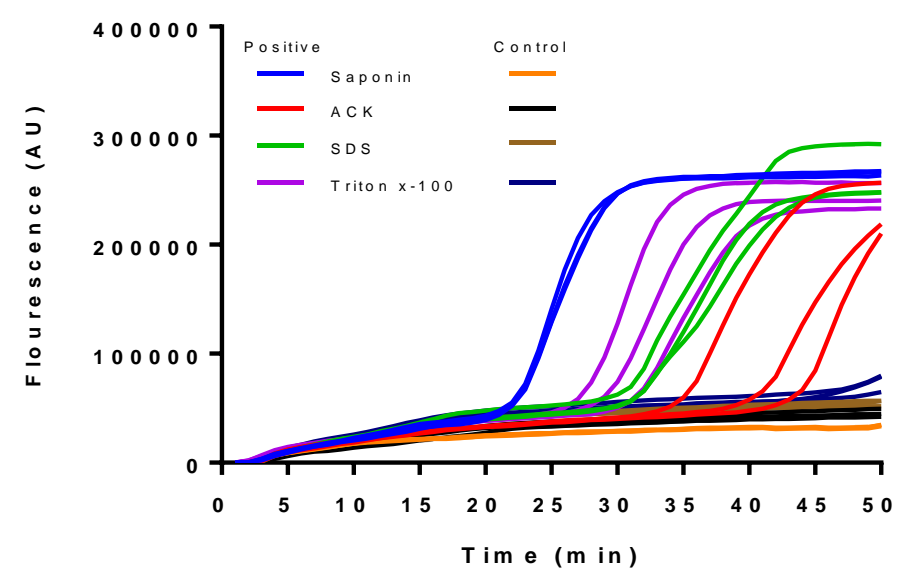

Fig S3. Baseline-subtracted raw fluorescence lysing composition characterization on $(n=3)$ samples of $1 \times 10^{3} \mathrm{cfu} / \mu \mathrm{l}$ E. coli in buffer.

a

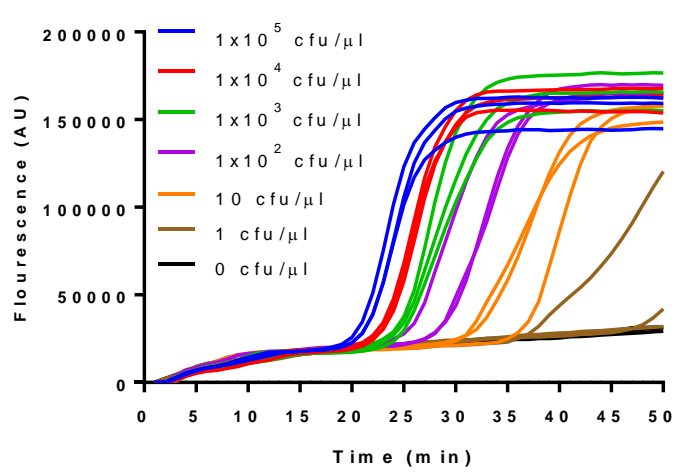

b

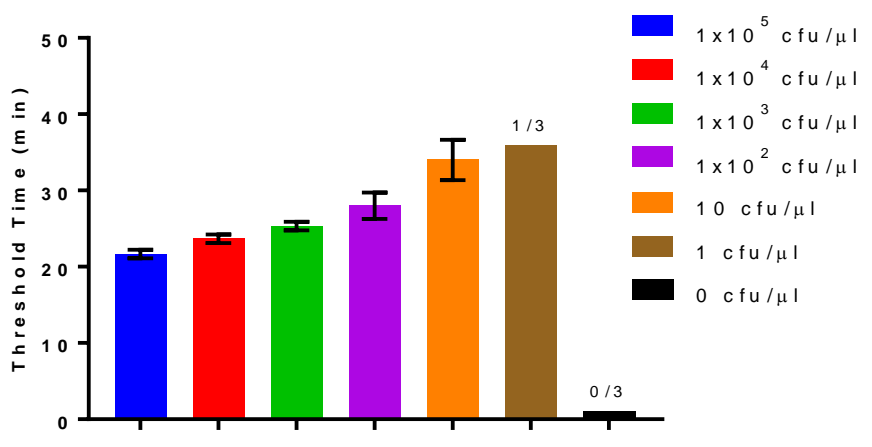

Fig S4. (a-b) Baseline-subtracted raw amplification curves and threshold plot for the detection of E. coli using saponin based lysing buffer. The detection limit is $10 \mathrm{cfu} / \mu \mathrm{L}$.

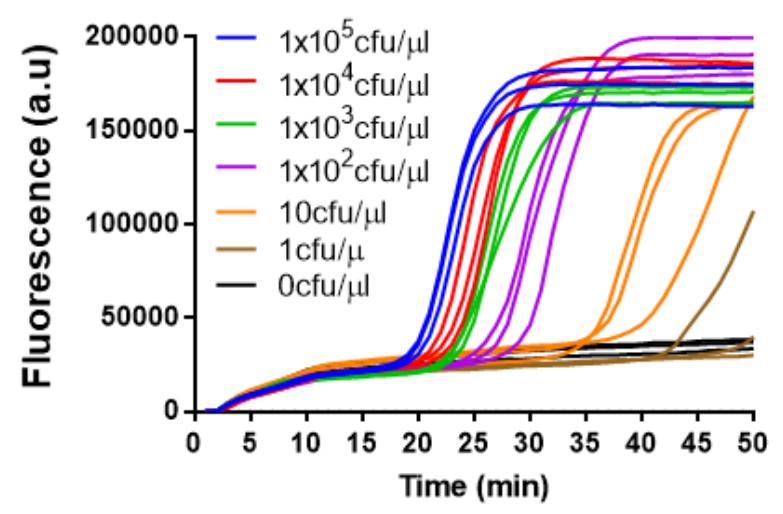

Fig S5. Baseline-subtracted raw amplification curves for the detection of E. coli using saponin based lysing buffer with $1 \times 10^{4} \mathrm{cfu} / \mathrm{\mu l}$ off target contamination in the sample for testing specificity. The assay maintains a detection limit of $10 \mathrm{cfu} / \mu \mathrm{L}$ with off target contamination. 
a

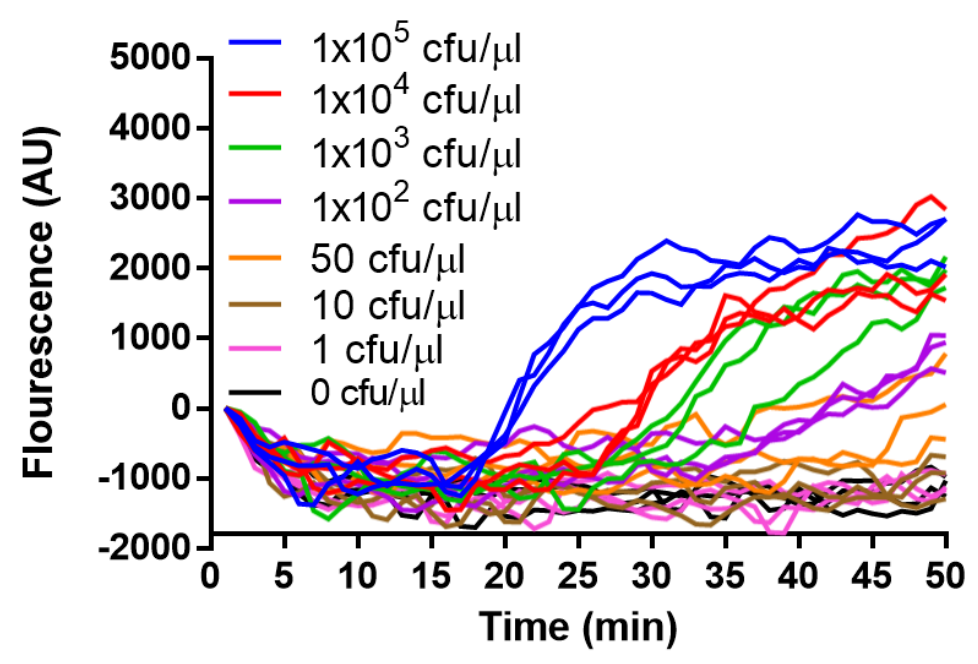

b

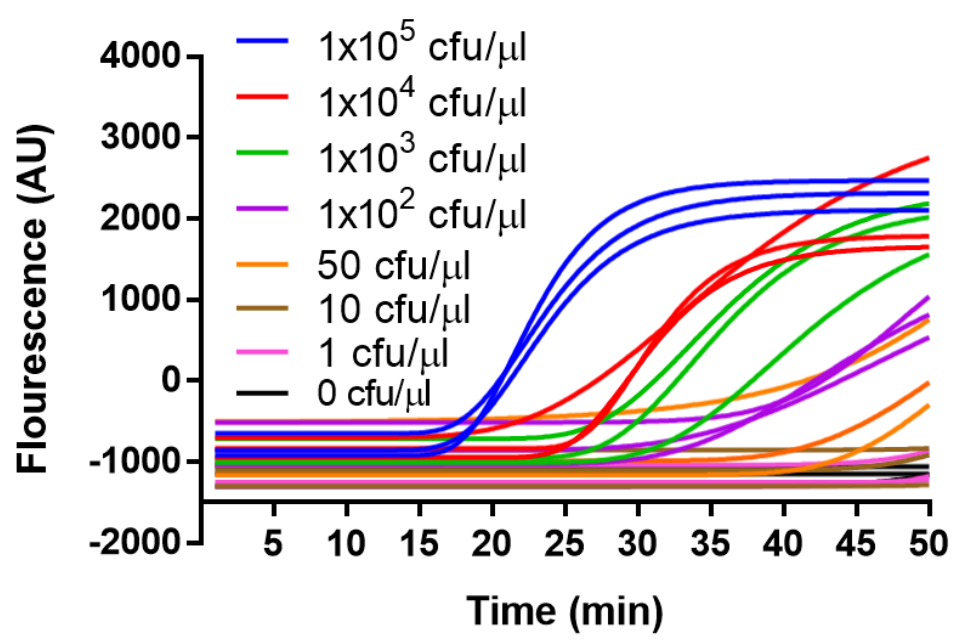

C

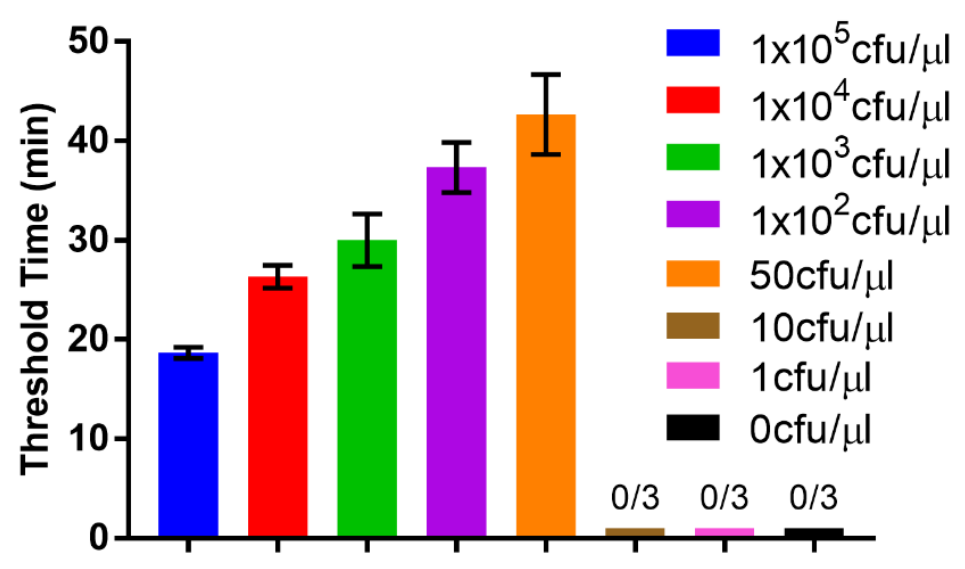

Fig S6. (a-c) Baseline-subtracted raw fluorescence amplification curves with sigmoidal fitting and threshold times $(n=3)$ for the detection of $E$. coli in whole blood samples. 

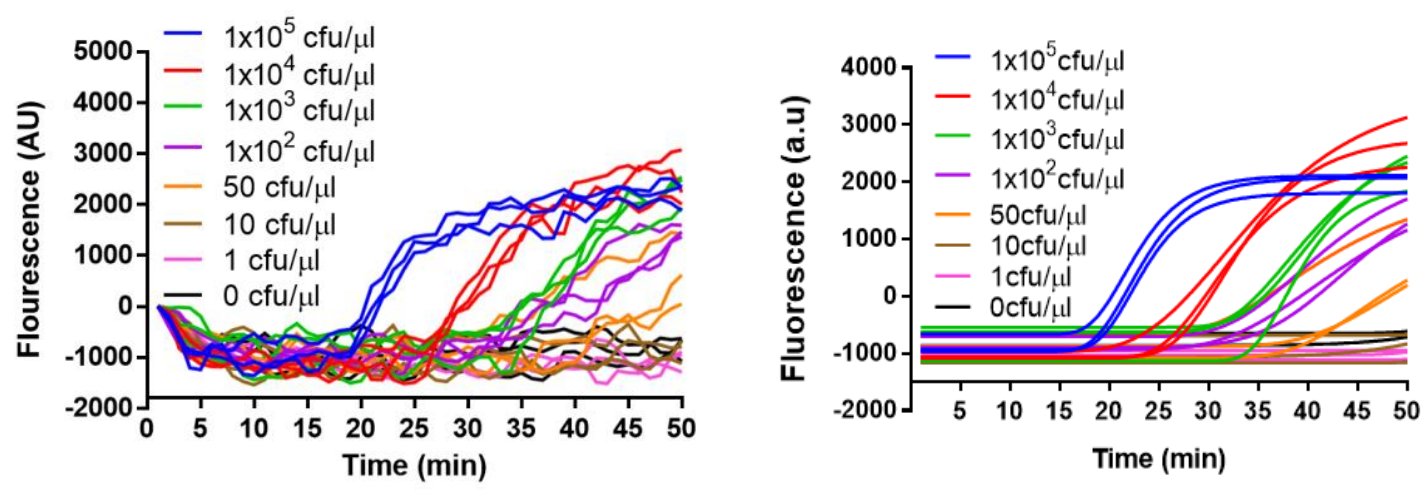

Fig S7. Baseline-subtracted fluorescence amplification curves with sigmoidal fitting for the detection of E. coli in whole blood samples with $1 \times 10^{4} \mathrm{cfu} / \mu \mathrm{l}$ off target contamination in the sample for testing specificity.

\section{AM Device fabrication and feature analysis extraction:}

We fabricated and measured 18 different test parts representing three print orientations, three microchannel depths, and two cartridge thicknesses. After manufacturing the resolution chips, the devices were rinsed with IPA (isopropyl alcohol), cured inside a UV-oven, and then visually inspected. Finally, the cartridge was washed in water and dried using pressurized air to remove dust particles and resin residue.

Feature extraction began by analyzing 500 cross-sectional views over the length of the microchannels for each device. Figure S8a show a 3D scan of the $3 \mathrm{~mm}$ thick chip with $400 \mu \mathrm{m}$-deep microchannels printed in "orthogonal print direction" and Fig. S8b shows one of the cross-sectional views along the device. For each 2D cross-sectional plane, the difference between the top and bottom layer and separation between two opposing walls were recorded to detect the measured microchannel depth and width. The measurements were then averaged to find the measured microchannel dimensions. Thus, the width and depth dimensions of each microchannel on the resolution chip were measured at 500 points, and the distribution was analyzed to determine manufacturing accuracy. 


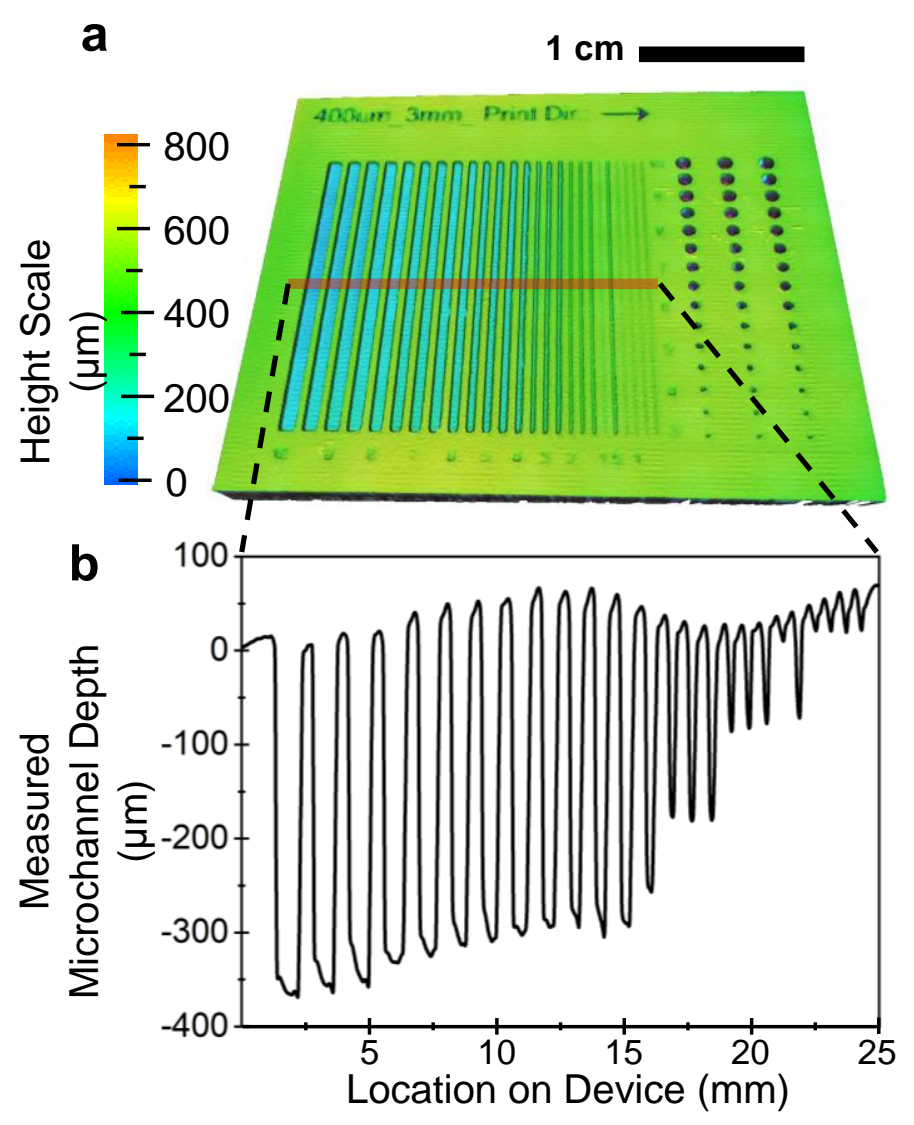

Fig S8. (a) Three-dimensional scan of $3 \mathrm{~mm}$ thick accuracy test part chip with $400 \mu \mathrm{m}$-deep microchannels device. (b) The middle cross-sectional view of the device. 
a

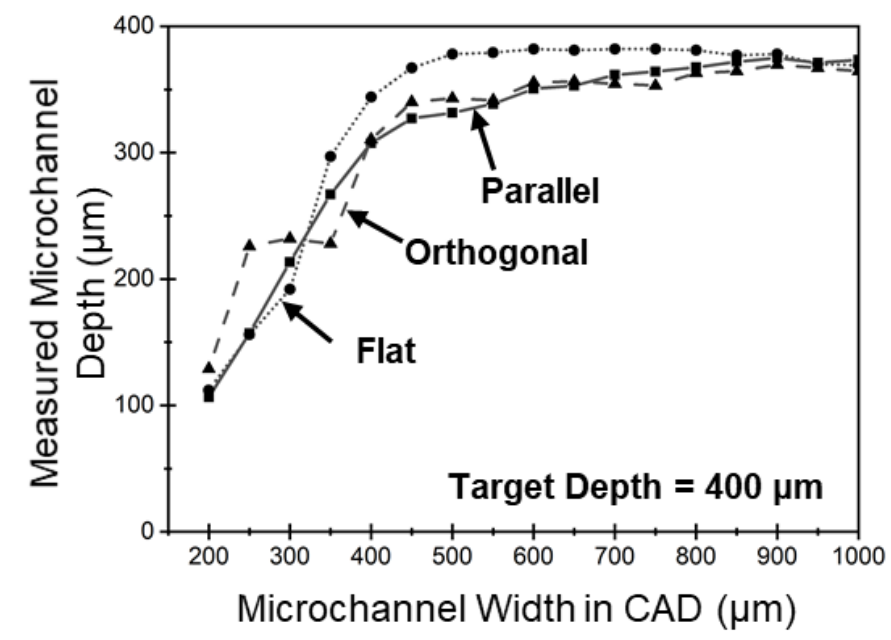

b

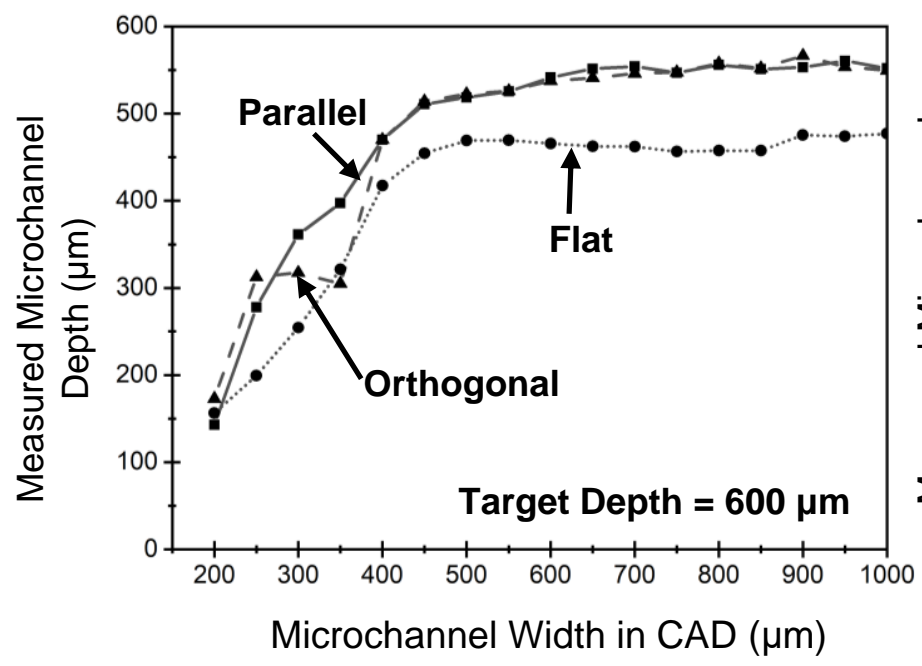

C

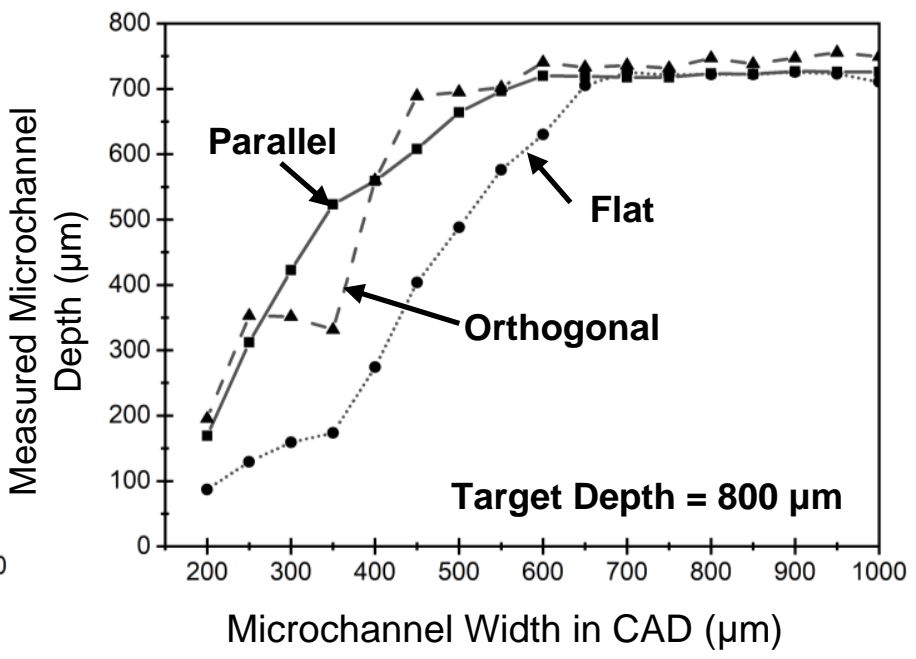

Fig S9. Process accuracy of microfluidic channels as a function of designed depth. Measured microchannel depth vs. designed microchannel width graphs for target depths of (a) $400 \mu \mathrm{m}$ (a) 600 $\mu \mathrm{m}$ and (c) $800 \mu \mathrm{m}$. 
a

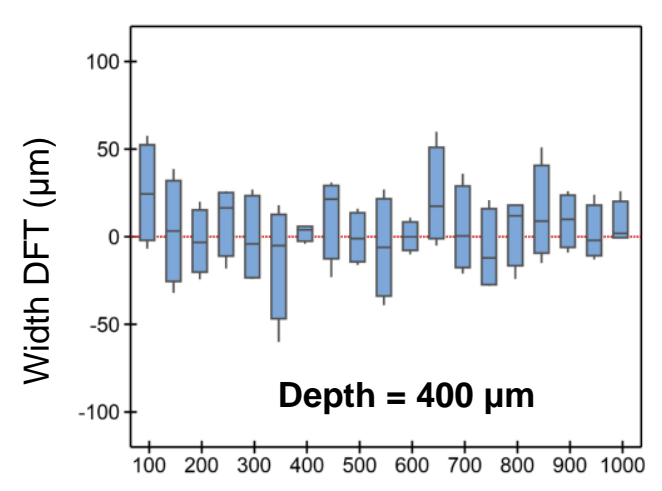

Microchannel Width in CAD $(\mu \mathrm{m})$

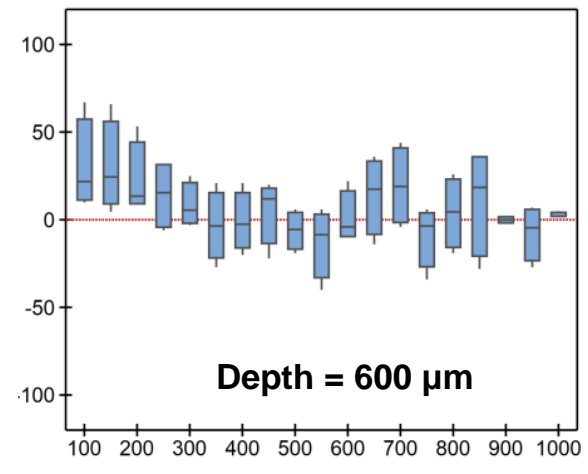

Microchannel Width in CAD $(\mu \mathrm{m})$

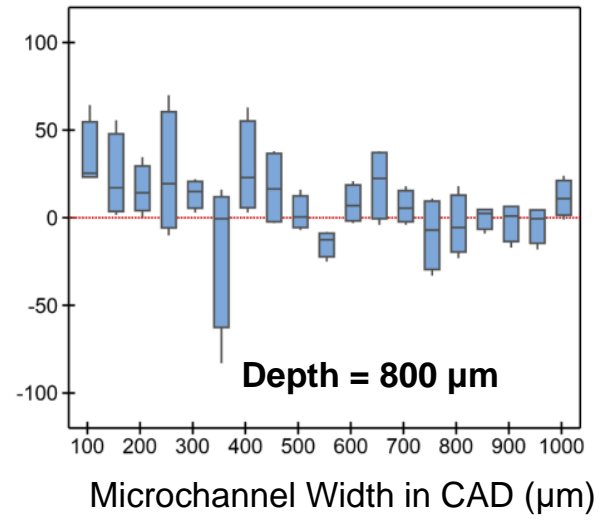

Microchannel Width in CAD $(\mu \mathrm{m})$

b
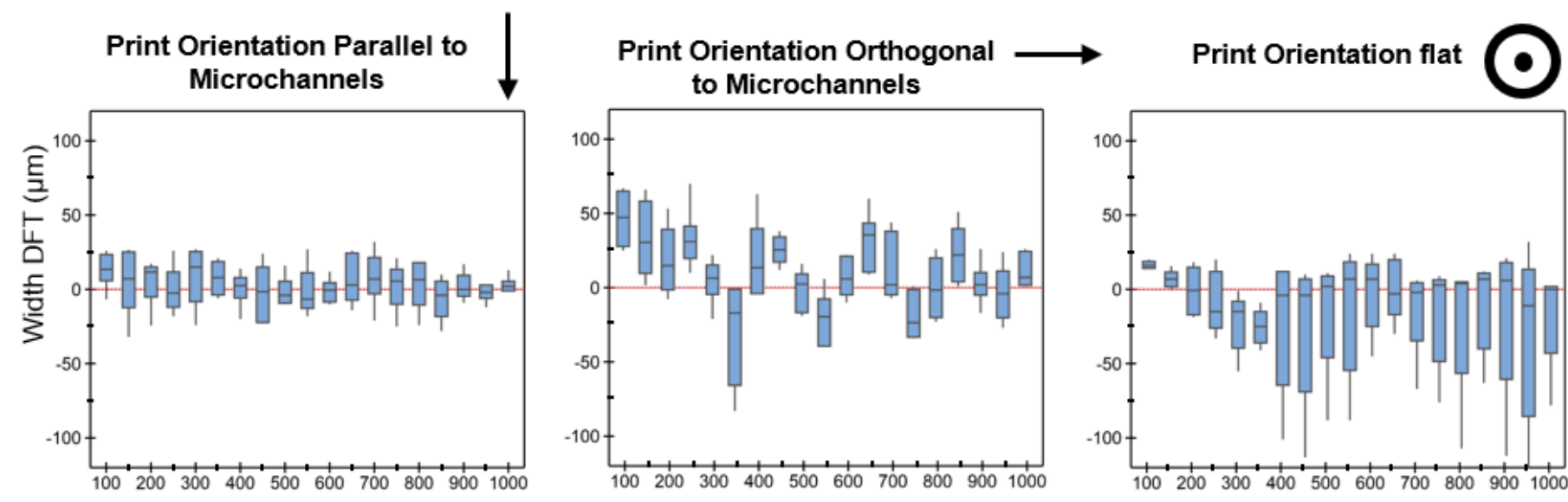

Fig S10. (a) Process accuracy for microfluidic channels as a function of designed width and designed depth. The data is shown in a box-plot format, where the interquartile range box represents the middle $50 \%$ of the data, and the median is indicated by the horizontal line in the box. The whiskers extending from either side of the box represent the maximum and minimum $25 \%$ of the data values. The difference from target (DFT) is the difference between width in CAD and measured width. Width DFT is shown as a function of width defined in CAD for target depths of $400 \mu \mathrm{m}, 600 \mu \mathrm{m}$, and $800 \mu \mathrm{m}$.

(b) Manufacturing Characterization for CLIP based AM microfluidics Process accuracy for microfluidic channels as a function of designed width and print orientation. The difference from target (DFT) is the difference between width in CAD and measured width. Width DFT is shown as a function of width defined in CAD for parallel, orthogonal, and flat print orientation. 
Table S1: Summary of manufacturing parameters by print orientation

\begin{tabular}{llll}
\hline Print Orientation & Devices Per Build & $\begin{array}{l}\text { Print Time } \\
\text { per Build [min] }\end{array}$ & $\begin{array}{l}\text { Production Rate } \\
\text { [Devices / min] }\end{array}$ \\
\hline Flat & 4 & 17 & 0.24 \\
Parallel & 46 & 222 & 0.21 \\
Orthogonal & 28 & 89 & 0.31 \\
\hline
\end{tabular}

The mean width DFT varies around zero for wide microchannels as opposed to narrow microchannels being printed consistently wider than target dimensions. The width DFT for narrower microchannels has about twice the standard deviation of the wide channels. When averaged over all width levels, the "parallel" print orientation has a mean DFT of $2.7 \mu \mathrm{m}$ and a standard deviation of $14.0 \mu \mathrm{m}$. In comparison, the "orthogonal" and "flat" print orientations have global means of 10.1 and $-12.2 \mu \mathrm{m}$ with standard deviations of 26.7 and $35.3 \mu \mathrm{m}$, respectively. We can conclude that "parallel" print orientation should be preferred over other print orientations for its higher printing resolution. 

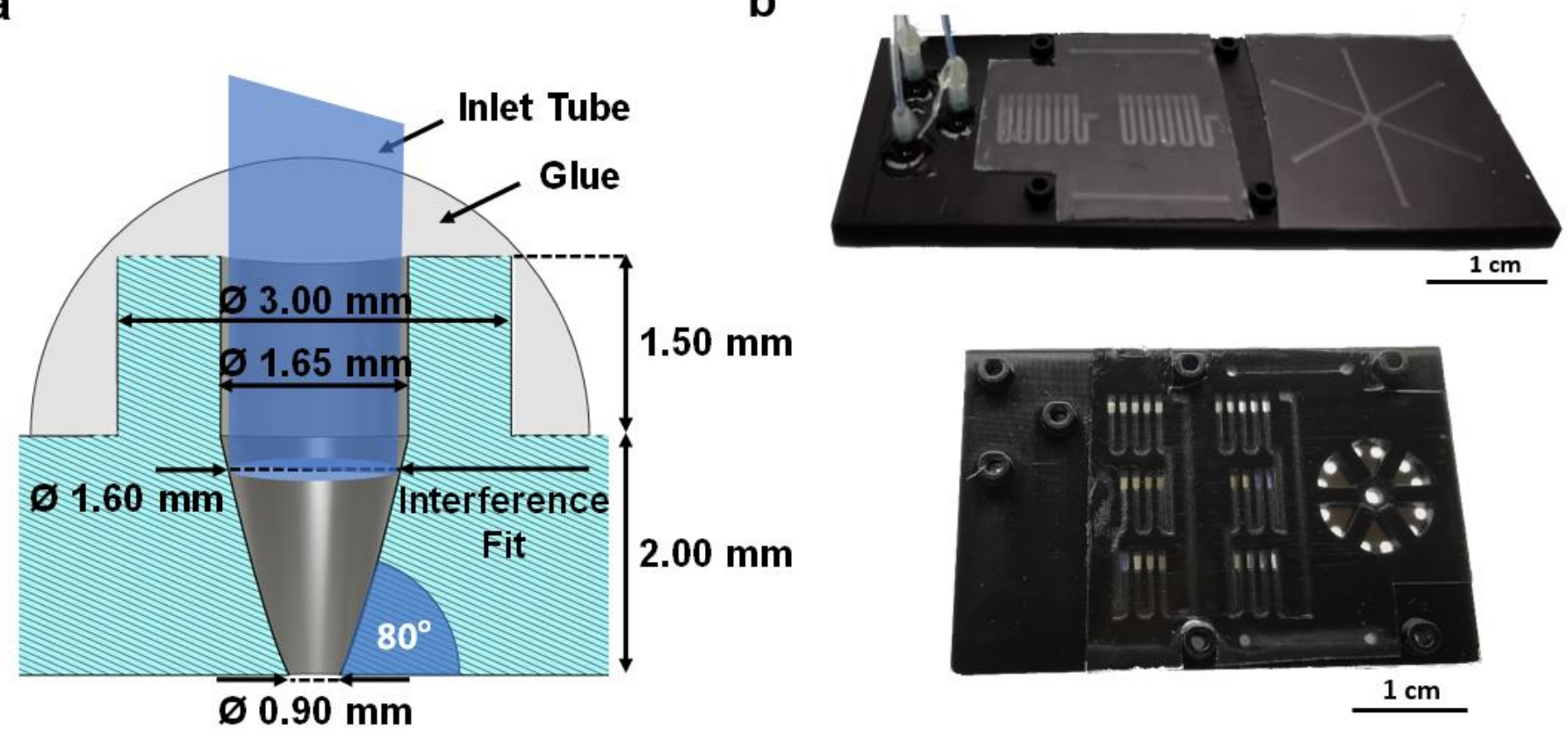

\section{$1.50 \mathrm{~mm}$}

Fig. S11. (a) Packaging of the diagnostic cartridge showing tapered inlet structure and interference fitting of tubes. The diameter of the hole for tubing gradually shrinks to establish an interference fit with the tube. Single control channels are loaded directly with a pipette, and do not require tubing. We tested a range of flow rates and pressures and found that this configuration can sustain pressure $>180 \mathrm{~Pa}$ and flow rates $\sim 30 \mu \mathrm{L} / \mathrm{min}$, which are sufficiently large for many applications. If a more robust seal is required, the tube holders can be alternatively covered by a UV-curable glue (Permabond UV632, Permabond Adhesives) and subsequently cured for 10 seconds using a UV-light source (IntelliRay 600, Uvitron). The UV-enhanced packaging endures a higher pressure of 6,500 $\mathrm{Pa}$ and a flow rate of up to $1,100 \mu \mathrm{L} / \mathrm{min}$. (b) Packaged cartridge designs prior to sample processing and amplification on chip.

\section{Design and characterization of a portable detection system}

Figure S12a-b shows the cross-sectional view of the smartphone-based reader detailing the components of the system and a photograph of the reader. The Portable detection system consists of a 3D printed cradle body to house the optical components for smartphone imaging and the smartphone. To characterize the illumination uniformity for the imaging area, we used an auto-fluorescent sheet with uniform emission properties, and measured the imaged pixel intensity in 9 regions, each with a 500x500 pixel area. The pixel intensity values per region, and region to region, show small deviation, indicating the LED arrangement and optical components provide uniform illumination (Fig. S12c-d). After optical characterization, we processed samples off-chip with lysing buffer and amplification reagents and subsequently loaded a CLIP based AM device with straight channels to determine material compatibility for amplification and imaging parameters (fig.S12e). This allowed us to determine the proper imaging parameters to distinguish amplified samples vs controls before translating all processing onto a single diagnostic module. We imaged channels loaded with positive and negative samples of blood and buffer with $1 \times 10^{4} \mathrm{cfu} / \mu \mathrm{l}$ samples. The portable detection system can distinguish with significance between positive amplified samples and negative controls on CLIP based AM devices from both blood and buffer using respective optimized imaging parameters (fig S12f-g). 
a

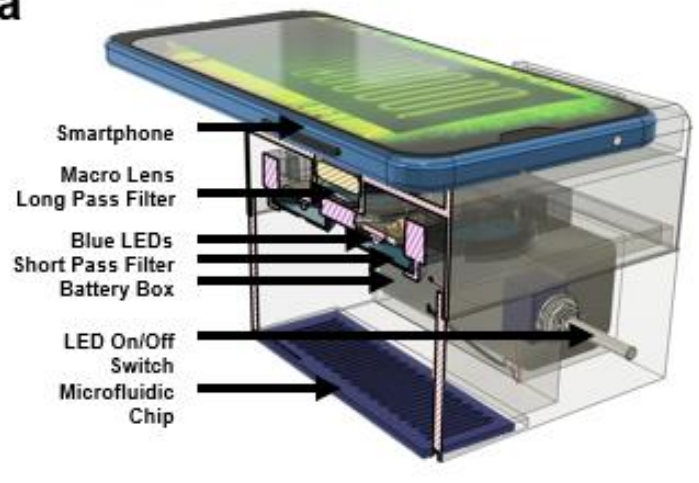

b

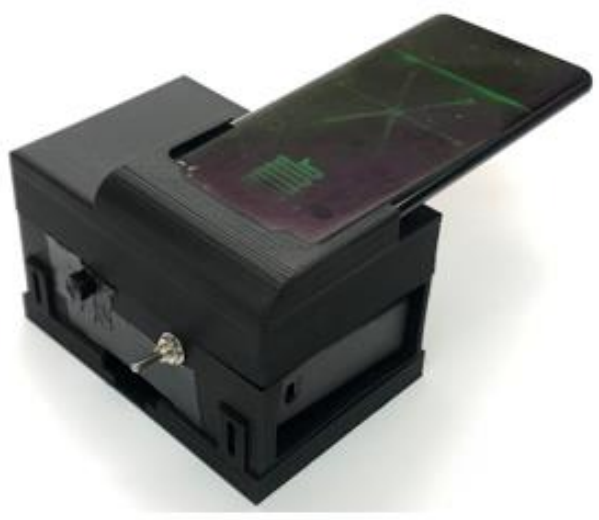

C

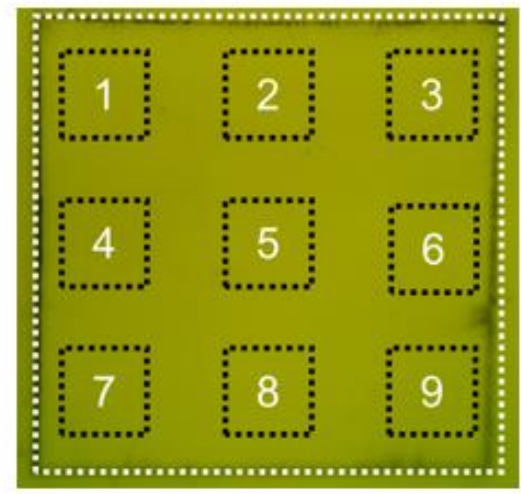

e

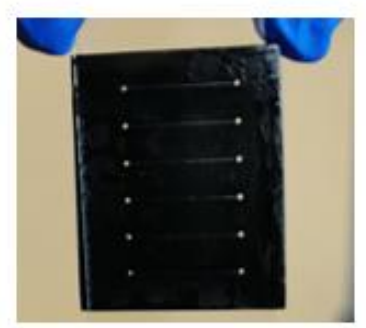

$\mathbf{f}$

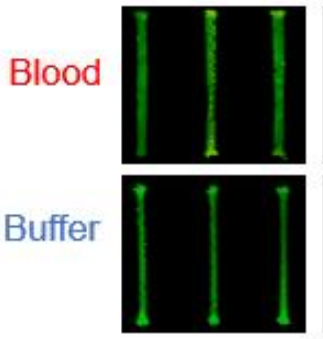

d

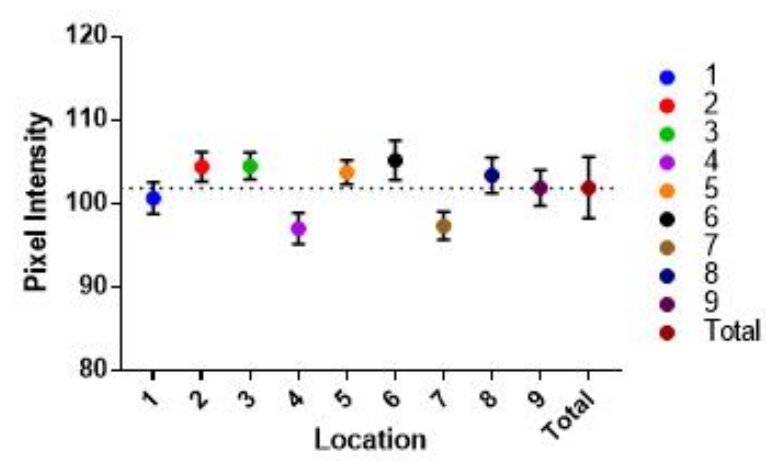

g

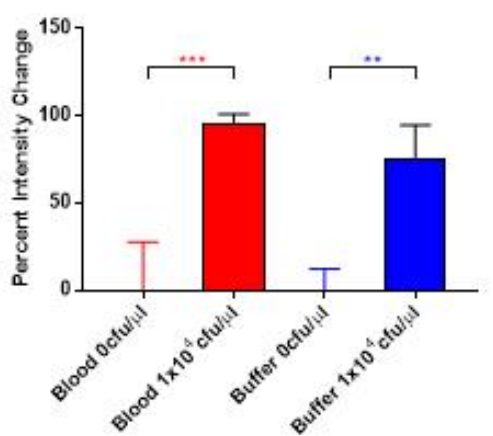

Fig S12. Portable detection system and imaging characterization for detection of E. coli. (a) Schematic of cradle components for portable imaging system. (b) Photograph of the portable detection system. Optical components integrated with the instrument are chosen to maximize the excitation and emission signal from the LED's and fluorescent signal, respectively. (c-d) a 1 inch $^{2}$ area from an auto fluorescent sheet is imaged and divided into nine sections of with a $500 \times 500$ pixel area characterized by the intensity measurements of each pixel per section. The optical instrumentation allows uniform illumination across the cartridge imaging area. (e) Photograph of straight channel control device. (f) Demonstration of amplification and detection of E. coli present in buffer and blood samples on CLIP based AM straight channel devices using the developed smartphone-based portable detection system. (g) Optimized imaging parameters allows differentiating intensity between positive and negative samples with significance. 